\title{
Crescent-shaped electron velocity distribution functions formed at the edges of plasma jets interacting with a tangential discontinuity
}

\author{
Gabriel Voitcu ${ }^{1}$ and Marius Echim ${ }^{1,2}$ \\ ${ }^{1}$ Institute of Space Science, Magurele, 077125, Romania \\ ${ }^{2}$ Belgian Institute of Space Aeronomy, Brussels, 1180, Belgium
}

Correspondence: Gabriel Voitcu (gabi@spacescience.ro)

Received: 7 September 2018 - Discussion started: 13 September 2018

Revised: 29 October 2018 - Accepted: 30 October 2018 - Published: 14 November 2018

\begin{abstract}
In this paper we discuss numerical simulations that illustrate a physical mechanism leading to the formation of crescent-shaped electron velocity distribution functions at the edges of a high-speed plasma jet impacting on a thin, steep and impenetrable tangential discontinuity with no magnetic shear. We use three-dimensional particle-in-cell simulations to compute the velocity distribution function of electrons in different areas of the plasma jet and at different phases of the interaction with the discontinuity. The simulation set-up corresponds to an idealized, yet relevant, magnetic configuration likely to be observed at the frontside magnetopause under the northward interplanetary magnetic field. The combined effect of the gradient-B drift and the remote sensing of large Larmor radius electrons leads to the formation of crescent-shaped electron velocity distribution functions. We provide examples of such distributions "measured" by a virtual satellite launched into the simulation domain.
\end{abstract}

\section{Introduction}

The terrestrial magnetosheath is often populated by highspeed plasma jets (also called plasmoids or plasma irregularities) streaming towards the frontside magnetopause (e.g. Dmitriev and Suvorova, 2012, 2015; Karlsson et al., 2012; Gunell et al., 2014) and impact it several times per hour (Plaschke et al., 2016). These finite-size plasma structures have an excess of anti-sunward dynamic pressure with respect to the ambient magnetosheath (e.g. Hietala et al., 2012; Savin et al., 2012; Archer and Horbury, 2013; Plaschke et al., 2013, 2017; Karlsson et al., 2018), and their interaction with the magnetopause can trigger local and global magne- tospheric and geophysical effects (e.g. Plaschke et al., 2009; Hietala et al., 2012, 2018). Dmitriev and Suvorova (2015) show that $>60 \%$ of the jets detected by the Time History of Events and Macroscale Interactions during Substorms (THEMIS) mission do penetrate inside the Earth's magnetosphere, while the rest of $<40 \%$ are not able to cross over the magnetopause. Some of the penetrating plasmoids were intersected in situ by spacecraft deep inside the magnetosphere (e.g. Lundin and Aparicio, 1982; Woch and Lundin, 1991, 1992; Yamauchi et al., 1993; Lu et al., 2004; Gunell et al., 2012; Shi et al., 2013; Lyatsky et al., 2016).

The transport of plasma jets, clouds and blobs across various magnetic field configurations has been investigated with magnetohydrodynamic (MHD) (e.g. Ma et al., 1991; Dai and Woodward, 1994, 1995, 1998; Huba, 1996), hybrid (e.g. Savoini et al., 1994; Palmroth et al., 2018) and kinetic (e.g. Galvez, 1987; Galvez et al., 1988; Livesey and Pritchett, 1989; Galvez and Borovsky, 1991; Cai and Buneman, 1992; Neubert et al., 1992; Hurtig et al., 2003; Gunell et al., 2009) numerical simulations (see also the review by Echim and Lemaire, 2000). These previous simulations did not reveal all the fundamental aspects of jet dynamics due to the inherent physical and/or geometrical limitations. For instance, the MHD and hybrid simulations do not quantify the effect of self-polarization (Schmidt, 1960), which is purely kinetic and plays a crucial role for plasma motion across transverse magnetic fields. On the other hand, the particle-in-cell (PIC) simulations were limited by the existing computing resources and none of them succeeded in evaluating the interaction of a three-dimensional plasma jet with a tangential discontinuity (TD). Recently, Voitcu and Echim $(2016,2017)$ used a 3D PIC code to simulate the dynamics of high-speed plasma 
jets interacting with magnetic fields typical for a tangential discontinuity with no shear. Such a configuration is similar to the magnetopause under a northward interplanetary magnetic field (IMF). It has been shown that the plasma-field interaction process is controlled by the dynamic and kinetic pressure of the high-speed jet, its electric polarizability and the magnetic jump at the TD. It was also shown that when the dynamic pressure is large enough the jet can cross the $\mathrm{TD}$, as implied by kinetic models like the impulsive penetration mechanism (Lemaire, 1977, 1985; see also Lemaire and Roth, 1991). When the dynamic pressure is small, the jet is stopped by the TD, consistent with THEMIS observations of jets reported by Dmitriev and Suvorova (2015). The dynamics of finite-size plasma jets and clouds across transverse magnetic fields have been also described with Vlasov equilibrium solutions by Echim and Lemaire (2005).

In this paper we discuss the kinetic structure of a low-beta plasma jet impacting on a thin, steep and impenetrable tangential discontinuity with no magnetic shear. The analysis is based on 3-D electromagnetic PIC simulations. We consider here an idealized, yet relevant, simulation set-up that corresponds to a magnetosheath plasma jet streaming towards the frontside region of the Earth's magnetopause and interacting with it under a northward interplanetary magnetic field. The plasma jet has a relatively small momentum and it cannot move across the rather steep discontinuity; it is repelled and split in two plasma streams moving in opposite directions, tangentially to the TD surface, as discussed in detail in a previous publication (Voitcu and Echim, 2017). However, kinetic processes observed during the interaction between the jet and the discontinuity lead to the formation of crescentshaped electron velocity distribution functions (VDFs).

Crescent-like VDFs were observed for ions in the Earth's magnetotail, close to the neutral sheet and plasma sheet boundary layer (e.g. Nakamura et al., 1991, 1992; Wilber et al., 2004), as well as in the magnetosheath, close to the magnetopause (e.g. Marcucci et al., 2004). The crescentshaped ion VDFs have been associated with various finite Larmor radius effects (e.g. Lee et al., 2004; Marcucci et al., 2004; Voitcu and Echim, 2012; Voitcu et al., 2012). Recently, crescent-shaped VDFs were observed by the Magnetospheric Multiscale (MMS) mission for electrons close to the dayside magnetopause (Burch et al., 2016) and were associated with meandering electron orbits near magnetic field reversal in the reconnection sites (Bessho et al., 2016; Lapenta et al., 2017). Egedal et al. (2016) proposed a two-dimensional theoretical model for the formation of crescent-shaped electron VDFs in asymmetric magnetic reconnection. The magnetic configuration used in our study does not involve the presence of magnetic field reversal regions, as in, for instance, Bessho et al. (2016), Egedal et al. (2016) or Lapenta et al. (2017), since we consider a tangential discontinuity with no shear across it. The physical mechanism revealed by our PIC simulations leads to crescent-shaped VDFs and is specific to the interaction between a plasma jet and a parallel non-uniform mag-

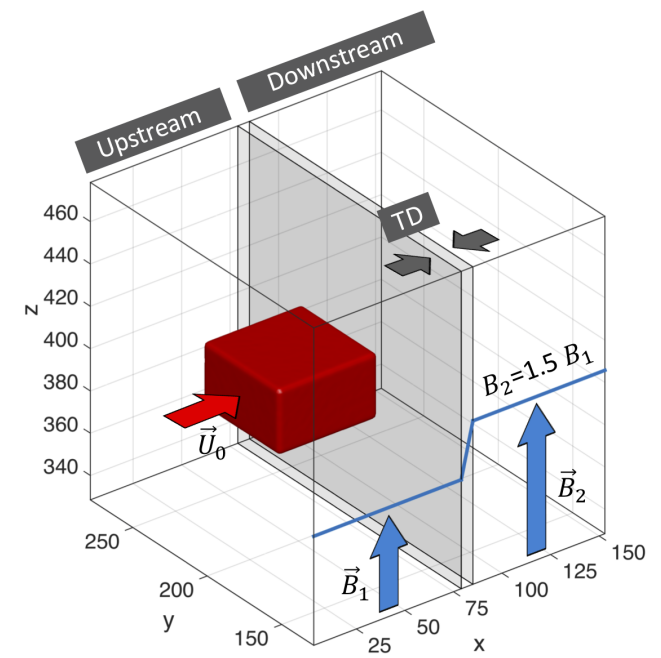

Figure 1. Schematic diagram of the simulation set-up. The highspeed plasma jet (shown by the red 3-D box) moves from the upstream region towards a parallel tangential discontinuity. The spatial profile of the background magnetic field is shown in blue. The tangential discontinuity is located between $x_{1}=79$ and $x_{2}=85$.

netic field; it does not require magnetic reconnection. This paper provides new insight into the kinetic structure of highspeed plasma jets moving in the vicinity of unidirectional magnetic barriers. We emphasize the role of finite Larmor radius effects that give rise to crescent-shaped electron distributions in the space of perpendicular velocities. Such electron VDFs can indicate the presence of a finite-size plasma jet deflected tangentially near a parallel magnetic discontinuity.

\section{Simulation set-up}

We consider a three-dimensional simulation geometry that allows the simultaneous investigation of plasma convection along the injection direction, the electrostatic selfpolarization along the normal to the magnetic field direction and the parallel expansion along the magnetic field lines. A schematic overview of the simulation set-up is given in Fig. 1. The finite-size plasma jet is illustrated by the red 3D box. We envisage the case of small Larmor radius plasma jets, i.e. the transversal dimension of the localized plasma structure is much larger than the ion Larmor radius.

The background magnetic field is parallel to $+O z$ everywhere inside the simulation domain. Its magnitude increases by $50 \%$ between two asymptotic states: $B_{1}$ (upstream, or left, of TD) and $B_{2}$ (downstream, or right, of TD). The tangential discontinuity is located between $x_{1}=79 \Delta x$ and $x_{2}=85 \Delta x$ (where $\Delta x$ is the simulation grid spacing). Its width is of the order of few ion Larmor radii, in agreement with theoretical arguments (e.g. Willis, 1978; Roth et al., 1996) and observations (e.g. Cluster measurements, see, for 
instance, Haaland et al., 2004) of the magnetopause thickness. Initially, the electric field is set to zero in the entire simulation domain. We assume that the background steadystate magnetic field is established prior to the injection of the plasma jet into the simulation domain and that this configuration remains unchanged during the entire simulation runtime. The self-consistent magnetic field computed during the simulation runtime adds to the background field. Thus, the simulated plasma jet can be seen as a perturbation of the equilibrium solution describing the TD's magnetic field. Our goal is to investigate the microphysics of the plasma jet-magnetic field interaction process in the vicinity of the tangential discontinuity. The study of TD's formation and stability is beyond the scope of our paper and shall be considered in our future simulations. Consequently, the background plasma is disregarded in the present work and the finite-size plasma jet is streaming in a vacuum towards the magnetic discontinuity, a situation similar to a magnetosheath jet characterized by an excess of dynamic pressure in a rarefied background plasma. The kinetic features discussed in the rest of the paper do not depend on the properties of the background plasma, but on the geometry of the jet and the magnetic and electric field profile.

The electrons and protons forming the 3-D plasma jet are uniformly distributed over a rectangular region localized at the left side of the TD where the magnetic field is uniform. Their initial VDF is a displaced Maxwellian with the average velocity parallel to the positive $x$ axis and perpendicular to the magnetic field direction: $U_{0}=1.2 V_{\mathrm{Ti}}$ (where $V_{\mathrm{Ti}}$ is the thermal velocity of protons). The electron-to-ion thermal speed ratio is $V_{\mathrm{Te}} / V_{\mathrm{Ti}}=30$. The velocities of both species are initialized according to their corresponding displaced Maxwellian distribution function. Thus, at the beginning of the simulation, the plasma jet is injected into the simulation domain with an initial bulk velocity pointing towards the discontinuity surface. The size of the simulation domain equals $155 \times 405 \times 805$ grid cells, while the initial size of the plasma jet is $50 \times 50 \times 30$ grid cells.

The jet's plasma has a small beta $(\beta=0.1$, including both dynamic and thermal plasma pressure; $\beta_{\mathrm{e}}=0.09$, including only electron thermal pressure) and a large dielectric constant $\varepsilon$. In the simulations discussed in this paper $\varepsilon=500$, where $\varepsilon=1+m_{\mathrm{i}} n /\left(\varepsilon_{0} B^{2}\right)=1+\left(\omega_{\mathrm{pi}} / \omega_{\mathrm{Li}}\right)^{2}$ is defined as in, for example, Chen (1974) and Lemaire (1985), with $\omega_{\mathrm{pi}}$ the ion plasma frequency and $\omega_{\mathrm{Li}}$ the ion Larmor frequency. Since $\varepsilon \gg 1$, collective dielectric effects play an important role in the dynamics of the plasma jet (Schmidt, 1960; Lemaire, 1985). In this case, a polarization electric field is formed inside the plasma jet that enables its convection across the transverse magnetic field (see Voitcu and Echim, 2016, for a detailed discussion on this topic). The ratio between the jet's transversal dimension and the ion Larmor radius is equal to 19. The total simulation time covers $4 T_{\mathrm{Li}}$ (where $T_{\mathrm{Li}}$ is the Larmor period of protons in the upstream region). This simulation configuration corresponds to the thin, steep and impen- etrable TD discussed in detail by Voitcu and Echim (2016, 2017).

The PIC code is based on TRISTAN (Buneman, 1993) and has been modified to simulate finite-size plasma structures (jets and plasmoids) interacting with non-uniform magnetic fields. The boundary conditions used are periodic for both particles and fields. In order to reduce the possible undesired effects of boundaries on the plasma dynamics, we keep the edges of the simulation domain as far as possible from the localized plasma jet. The total number of simulated particles is 30 million for each of the two plasma species, while the number of particles per simulation grid cell at initialization is equal to 400 per species. The proton-to-electron mass ratio is 36 . A detailed description of the PIC code is given by Voitcu (2014).

The velocity distribution function, $f$, at different time instances, is computed by using a uniform rectangular grid of spatial bins that covers the entire plasma jet. For each spatial bin, we calculate histograms by counting the number of particles, $N_{t}$, in each cell of a uniform rectangular grid defined in the velocity space. Thus, the VDF at time $t$, for a bin of width $(\delta x, \delta y, \delta z)$ in configuration space and a cell of width $\left(\delta v_{x}, \delta v_{y}, \delta v_{z}\right)$ in velocity space, is obtained by dividing $N_{t}$ to the volume of each 6-D cell in the phase space. The dimensions of the spatial bins and velocity cells are chosen such that the 6-D phase space is well sampled to provide a good representation of the VDF.

The physical quantities are normalized as follows: the number density is normalized to its initial value $n_{0}$, the particle velocities are normalized to their initial thermal speed $V_{T_{0}}=\left(2 k_{B} T_{0} / m\right)^{1 / 2}$. The VDF is normalized to the maximum value of the initial displaced Maxwellian distribution, $f_{0}=n_{0} /\left(\pi^{3 / 2} V_{T_{0}}^{3}\right)$. The time and spatial coordinates are normalized to the initial ion Larmor period $T_{\mathrm{Li}}$ and the grid spacing $\Delta x=0.5 r_{\mathrm{Le}}$.

\section{Numerical results}

The dynamics of the jet during its interaction with the TD was discussed by Voitcu and Echim $(2016,2017)$. When the TD is very steep, the jet is repelled and undergoes two dynamical phases: (A) the jet's frontside interacts directly with the non-uniform magnetic field of the TD, leading to vanishing of the self-polarization electric field, and (B) the jet is repelled and split into two counter-streaming plasma flows tangential to the TD surface. Here we focus on the kinetic properties of electrons of the deflected plasma jet and consider two instances of its dynamics, typical for phase A (illustrated by Fig. 2) and phase B (illustrated by Fig. 3), respectively.

We define a uniform grid in configuration space whose cells have the perpendicular size equal to $\delta x=6\left(\approx 3 r_{\mathrm{Le}}\right)$ and $\delta y=13\left(\approx 6 r_{\mathrm{Le}}\right)$, respectively. This spatial resolution is chosen in order to achieve a sampling of the numerical 


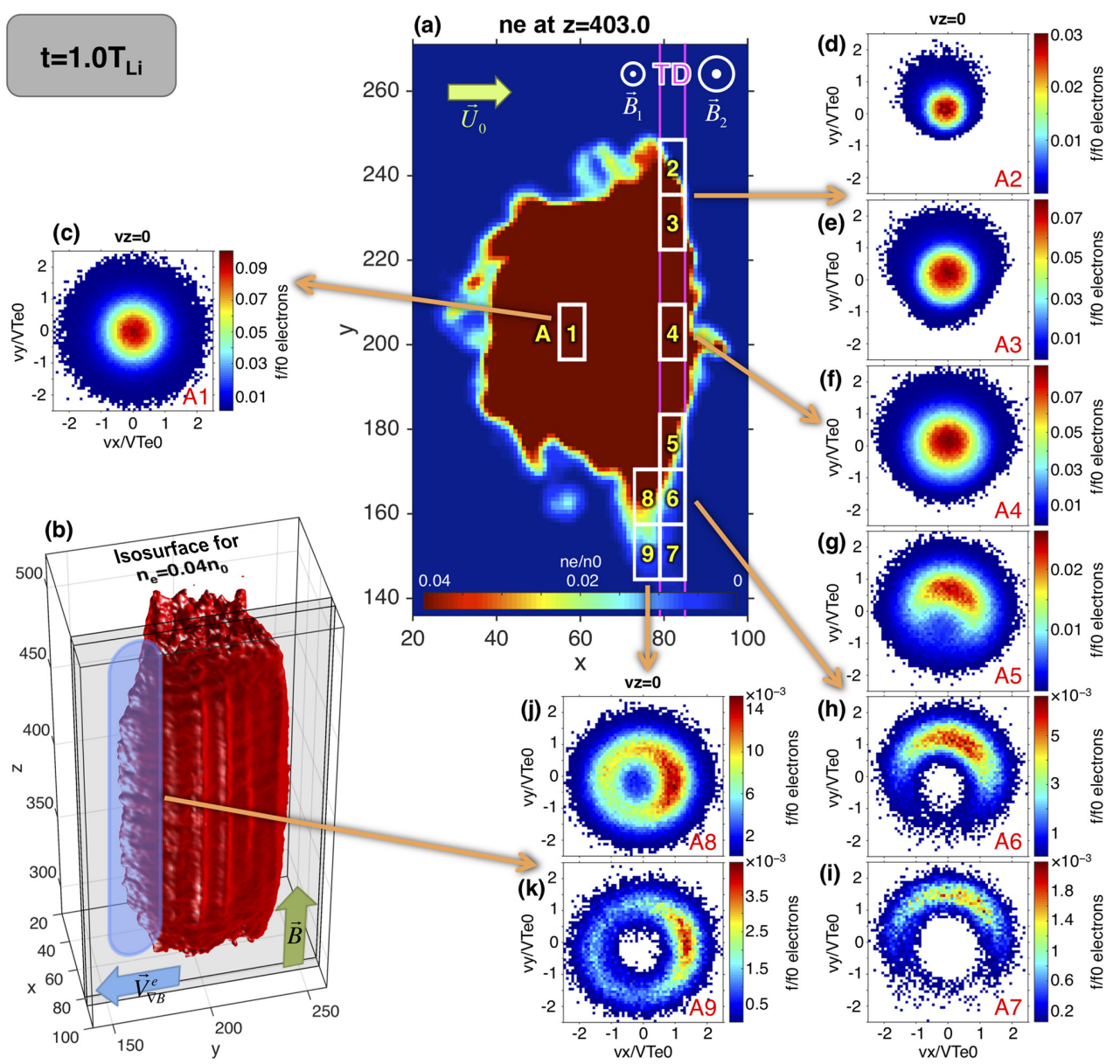

Figure 2. Snapshot of 3-D PIC simulation at $t=1 T_{\mathrm{Li}}$ (phase A). (a) Electron number density in the $x O y$ plane for $z=403$. (b) The 3-D density isosurface for $n_{e}=0.04 n_{0}$. (c-k) Electron VDFs in the space of perpendicular velocities for the spatial bins shown with white rectangles in panel (a). The two magenta lines mark the TD's boundaries.

VDFs comparable to the experimental data obtained with a state-of-the-art electron spectrometer in the vicinity of the magnetopause, like for example those on MMS whose time resolution is $30 \mathrm{~ms}$. The distance covered by MMS during $30 \mathrm{~ms}$ within a plasma flow of $100 \mathrm{~km} \mathrm{~s}^{-1}$ is of the order of few electron Larmor radii. In order to increase the number of particles collected in each bin of the spatial grid, the grid cells are expanded over the entire column of the simulation domain along the $z$ axis $(\delta z=800)$. Nevertheless, we performed tests for smaller $\delta z$ (not shown) and found that the relevant features of the VDF do not depend on the cell size along $O z$. A uniform 3-D grid is also defined in the velocity space whose cells dimensions are $\delta v_{z}=0.6 V_{\mathrm{Te}_{0}}$ and $\delta v_{x}=\delta v_{y}=0.08 V_{\mathrm{Te}_{0}}$. In the following we discuss sections of VDFs in the plane perpendicular to the magnetic field obtained from velocity cells whose centres are located in $v_{z}=0$.

The numerical simulations results obtained for the interaction of the high-speed plasma jet with the TD are shown in Fig. 2 (for $t=1 T_{\mathrm{Li}}$, corresponding to phase A) and Fig. 3 (for $t=1.9 T_{\mathrm{Li}}$, corresponding to phase $\mathrm{B}$ ). In panel (a) we illustrate the number density of electrons for the central cross section localized in $z=403$; panel (b) shows the 3-D shape of the plasma jet computed as the isosurface corresponding to $n_{e}=0.04 n_{0}$ in Fig. 2 and $n_{e}=0.02 n_{0}$ in Fig. 3. The VDF is calculated for 88 spatial bins that cover the entire jet at $t=1 T_{\mathrm{Li}}$ and 84 ones at $t=1.9 T_{\mathrm{Li}}$. We selected the most relevant nine bins, labelled A1-A9 in Fig. 2a and B1-B9 in Fig. 3a. The corresponding electron VDFs are shown in panels c-k of both Figs. 2 and 3. The VDF maps for the entire spatial grids are provided in Appendix A.

The electron VDF in the core of the plasma jet is a displaced Maxwellian similar to the initial one, as can be seen in Figs. $2 \mathrm{c}$ and $3 \mathrm{c}$. However, the VDFs at the boundaries of the deflected plasma jet are non-gyrotropic, as shown by bins A5-A9 and B5-B9, where the phase-space density is virtually empty in the central region, for small perpendicular velocities. The VDF takes, however, larger values in the sector 


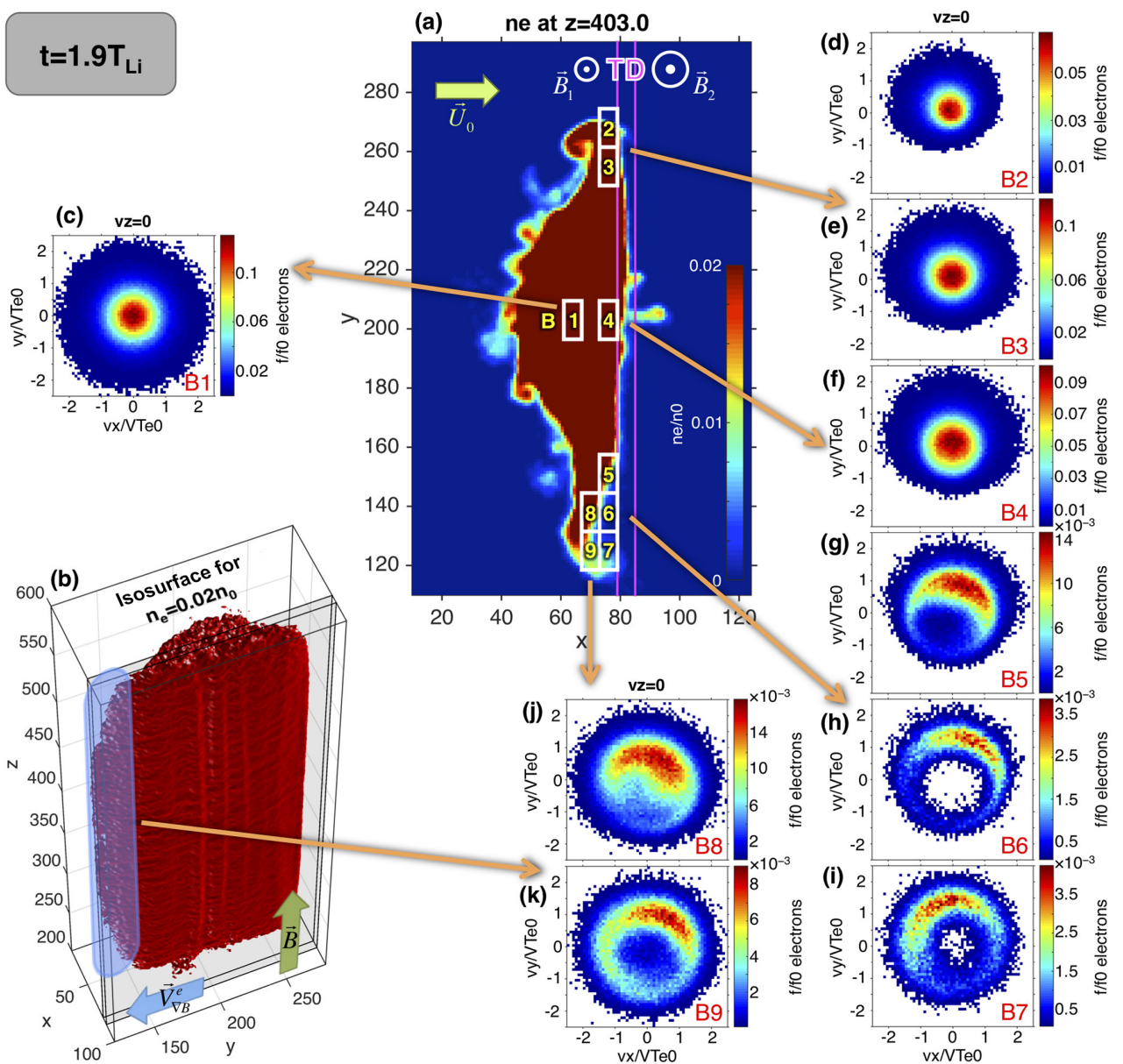

Figure 3. Snapshot of 3-D PIC simulation at $t=1.9 T_{\mathrm{Li}}$ (phase B). (a) Electron number density in the $x O y$ plane for $z=403$. (b) The 3$\mathrm{D}$ density isosurface for $n_{e}=0.02 n_{0}$. (c-k) Electron VDFs in the space of perpendicular velocities for the spatial bins shown with white rectangles in panel (a). The two magenta lines mark the TD's boundaries.

of a ring around the depleted core. These features give the VDF a crescent-like aspect. On the other hand, the VDFs in the frontal bins A2, A3, B2 and B3 are depleted at larger velocities, while the phase-space density for small velocities is large.

These non-gyrotropic features of the VDFs are observed in the plane of perpendicular velocities, inside the TD (bins A5-A7), but also outside it (bins A8, A9 and B5-B9). Their characteristics change with the distance from the jet boundaries. On the one hand, the size of the VDF's central cavity increases towards the lateral edge of the jet (compare, for instance, the VDFs plotted for bins A6 and A7). On the other hand, the VDFs take non-vanishing values only in limited sectors surrounding the central cavity; the peak VDF density in the ring varies from one bin to another (compare, for instance, the VDFs for bins A6 and A8 in Fig. 2).

The physical mechanisms contributing to the formation of crescent-shaped electron VDFs are illustrated by the diagrams in Fig. 4. The electrons in the frontside of the jet interact with the magnetic field gradient inside the discontinuity and drift in the - $O y$ direction with a velocity proportional to their gyration energy (Northrop, 1963). Thus, the electrons with higher kinetic energies are scattered to larger distances than the less energetic ones. The forward motion of the jet towards the discontinuity continuously replenish the electron population at the front edge. The gradient-B drift acts as a filter of the perpendicular velocities whose effect is the formation of an energy-dispersed structure in the frontal region of the plasma jet; the electrons with higher kinetic energies accumulate towards the jet's lateral edge, in the direction $-\boldsymbol{B} \times \nabla B$ (compare, for instance, Fig. $4 \mathrm{~b}$ and e). Due to this filtering effect, the regions far from the centre of the jet, along - $O y$, cannot be reached by the low-energy electrons whose gradient-B drift velocity is small. Thus, far from the jet's centre, the phase space of small velocities is empty and a central cavity is formed, as shown in Fig. $4 \mathrm{~d}$ and e. The VDF is therefore ring-shaped. The corresponding low-energy cutoff of the phase-space ring increases with the distance from 


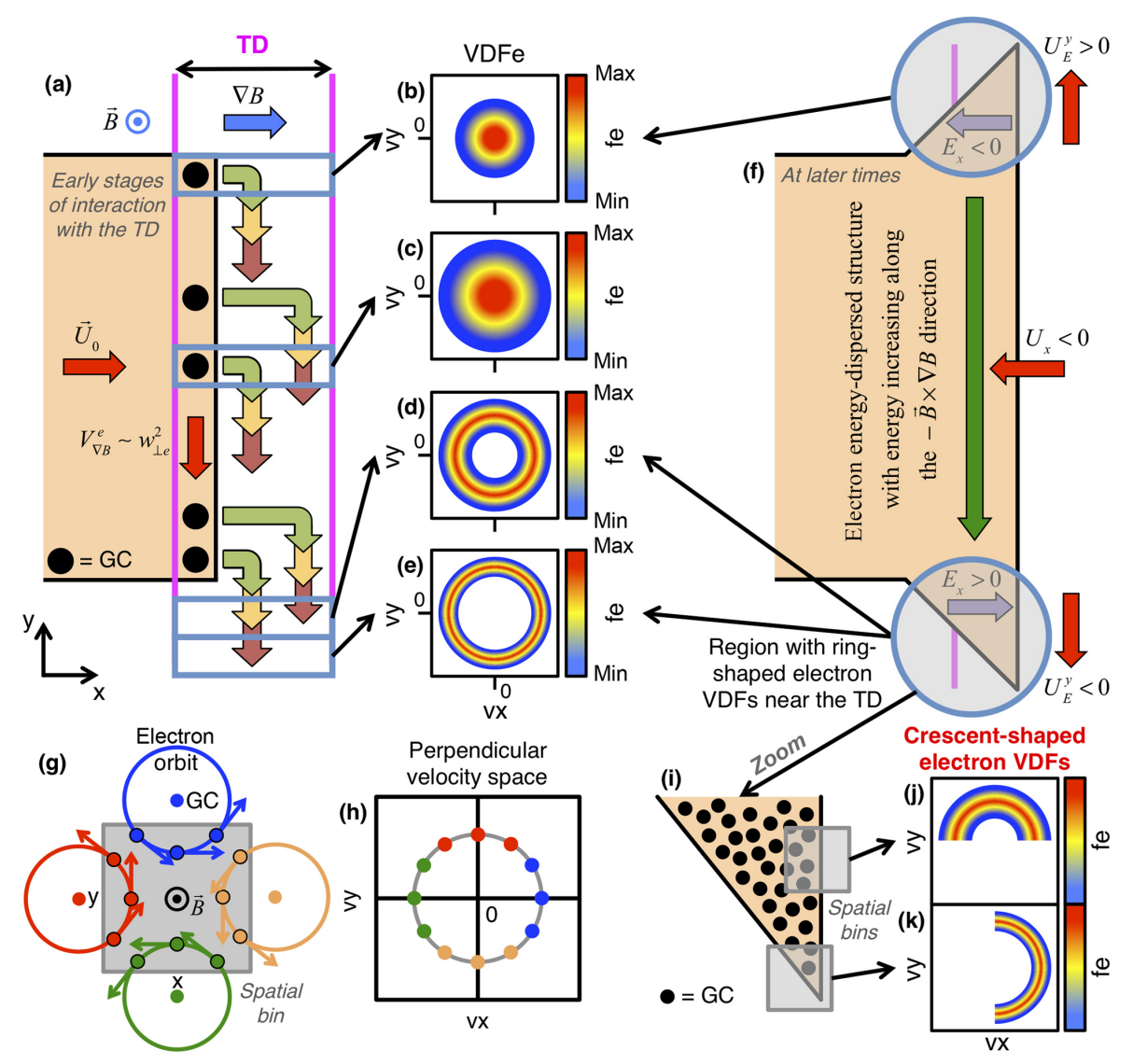

Figure 4. Panel (a) illustrates the phase A of plasma jet's interaction with the magnetic discontinuity. The black dots indicate the guiding centres (GCs) of electrons localized at the front edge. The multiple groups of green-yellow-red arrows show the gradient-B drift displacement of the GCs proportional to the gyration energy (green corresponds to the lowest energy). The four blue boxes indicate the bins where the VDF is computed. The electron VDFs obtained for the upper edge bins $(\mathbf{b}, \mathbf{c})$ are gyrotropic and their phase density vanishes for large velocities; the VDFs obtained for the bottom edge bins (d, e) are ring-shaped. Panels (g) and (h) illustrate how the electrons with GCs outside a spatial bin contribute to different sectors of the VDF collected in the respective bin. The colours of the dots in panel (h) identify which particle shown in panel (g) contributes to that sector of the VDF. This effect corresponds to a remote sensing of the large Larmor radius electrons outside the bin. Panels (f) and (i)-(k) show how the remote sensing effect contributes to the formation of crescent-shaped VDFs in a configuration similar to the PIC simulations.

the jet's centre along - $O y$, since the spatial regions increasingly farther from the central region of the jet are accessible to electrons with increasingly larger gyration velocities (compare the VDF's central cavities in Fig. 4d and e). Figure 2 and the diagrams in Fig. 4a-e show the formation of ring-shaped VDFs during phase A of the interaction between the jet and the TD.

Nevertheless, the kinetic features imprinted by the gradient-B drift during phase $\mathrm{A}$ are persistent and the nonMaxwellian electron VDFs are observed later in the simulation when the jet is pushed back, outside the TD. Indeed, during the backward convection of jet's frontal regions, the energy-dispersed structure is displaced in the $-O x$ direction. This explains the shape of VDFs in spatial bins localized in regions of uniform magnetic field, like A8 and A9 in Fig. 2 and B5-B9 in Fig. 3. Thus, the ring-shaped VDFs persist when the plasma drifts far from the actual interaction with the non-uniform field. The tangential deflection of the jet carries the energy-dispersed structure away from the impact zone and enhances the asymmetry of the lateral edges. The lowenergy electrons and the ring-shaped VDFs are displaced in opposite directions, to larger and larger distances from the core. The diagram in Fig. $4 \mathrm{f}$ illustrates this effect. Note that this mechanism partially explains the simulation results, but it does not account for the non-gyrotropy. To clarify this issue, we must consider a supplemental effect.

Let us discuss the VDFs obtained in spatial bins A5-A9 and B5-B9 from Figs. 2 and 3, respectively. The diagram in Fig. 4g illustrates such a rectangular bin, as well as the guiding centres of four electron orbits localized outside the bin's boundaries, whose Larmor radii are comparable to the size of the bin. Each orbit intersects the area of the bin for a lim- 
ited time interval and is counted when computing the VDF value in the velocity space region determined by the electron gyration velocity along the segment of the orbit inside the bin. That region in the velocity space changes for each of the four illustrative orbits considered in Fig. $4 \mathrm{~g}$ according to the gyration phase. For instance, within the segment localized in the bin, the gyration velocity of the electron following the red orbit takes values that are restricted to the upper sector of the perpendicular velocity space where $v_{y}>0$, while the gyration velocity of the electron along the blue orbit is restricted to the $v_{x}>0$ sector, as shown in Fig. 4h. The absence of certain guiding centres (and of their intersecting orbits) around the spatial bin decreases the VDF's phase density for the velocities corresponding to the missing guiding centres. Thus, the spatial inhomogeneity at the borders of the jet manifests as phase-space non-gyrotropy of the distribution function. This effect leads to the formation of non-gyrotropic VDFs in the spatial bin itself and is noticeable in regions of configuration space characterized by sharp variations in the density of particles with large kinetic energy.

The mechanism discussed above can be verified for a configuration similar to our numerical simulations. For this purpose, a schematic diagram is sketched in Fig. $4 \mathrm{i}$, where we consider two spatial bins close to the jet's boundaries. We discuss the VDFs collected in these two bins. The distribution of the guiding centres around the bins is non-uniform. Indeed, the top bin in Fig. $4 \mathrm{i}$ is predominantly intersected by large Larmor radius orbits whose guiding centres are found at the left side (like the red orbit in Fig. 4g), while the bottom bin is mostly crossed by orbits from above (similarly to the blue orbit in Fig. 4g). Therefore, the phase-space density of the VDF in the two spatial bins is non-vanishing only in a limited sector defined by $v_{y}>0$ (the top bin) and $v_{x}>0$ (the bottom bin), respectively. Thus, only the upper (right) part of the ring-shaped VDF in Fig. 4d (Fig. 4e) is accessible to the top (bottom) bin and the VDFs in these regions are crescent-shaped, as illustrated in Fig. $4 \mathrm{j}$ and k. This explains the results of our simulations. Indeed, if one checks the electron VDFs from the numerical simulations in Fig. 2, one notes the crescent-like features in bins A6 and A8 in Fig. 2a similar to the top and bottom bins in Fig. 4i (compare the VDFs in Fig. $2 \mathrm{~h}$ and $\mathrm{j}$ to the ones in Fig. $4 \mathrm{j}$ and $\mathrm{k}$ ). On the other hand, bin B5 in Fig. 3a is crossed by orbits that have the guiding centres localized at its left side and also above it. The crescent-shaped VDF in this case is rotated towards the $\left(v_{x}>0, v_{y}>0\right)$ sector of the perpendicular velocity space, as can be seen in Fig. 3g. Similar arguments explain the orientation of all crescent-shaped VDFs in Figs. 2 and 3.

The discussion above shows that the formation of crescentlike electron VDFs at the edges of a low-beta plasma jet interacting with a magnetic discontinuity is the result of a combined effect due to (i) the formation of an energy dispersion region at the front edge of the plasma jet sustained by the energy-dependent gradient-B drift - this effect leads to the formation of a ring shaped VDF - and (ii) the remote sens- ing of large Larmor radius electrons at the lateral edge of the jet - this effect explains why certain regions of the ringshaped VDFs have very small phase density, leading to the crescent-like shape. The simulations also show that the kinetic structure resulting from the interaction with the discontinuity is maintained at later stages, when the jet is deflected and pushed outside the TD. Indeed, the non-gyrotropic features imprinted by the interaction of the low-beta plasma jet with the steep tangential discontinuity are observed during its subsequent motion. In the case of high-beta plasma jets, we expect that the dynamics of the electrons and ions to be affected by the magnetic perturbation produced by the jet itself and, consequently, to alter the velocity filtering and remote sensing effects, leading to the formation of crescent-shaped electron VDFs. Supplemental simulations are envisaged in the future to clarify this aspect. It should be mentioned that the ion VDFs (not discussed here) do not show any crescent features in the perpendicular velocity space.

Numerous recent spacecraft observations show the omnipresence of magnetosheath jets and therefore there is an increased interest in their kinetic structure (see for example Plaschke et al., 2017; Karlsson et al., 2018). Below we discuss a possible method to check the results of our simulations against spacecraft observations. For this purpose, we "launched" a virtual satellite into the simulation domain. The VDF is "measured" by counting the particles localized within a fixed spatial bin centred on the virtual satellite. We use the same spatial resolution as in Figs. 2 and 3, and thus the resolution of the virtual satellite measurements is comparable to the very high-resolution electron measurements provided by MMS. In Fig. 5 we show five examples of electron VDFs measured by the virtual satellite. For consistency, we consider the same satellite S1 as in Voitcu and Echim (2017). A virtual time series of VDFs is collected at $x=75, y=153$ and $z=403$, at $t_{1}=1.03 T_{\mathrm{Li}}, t_{2}=1.54 T_{\mathrm{Li}}$, $t_{3}=2.05 T_{\mathrm{Li}}, t_{4}=2.57 T_{\mathrm{Li}}$ and $t_{5}=3.08 T_{\mathrm{Li}}$. We illustrate the perpendicular cross sections corresponding to $v_{z}=0$ and use the same resolution in velocity space as in Figs. 2 and 3. The temporal separation of the five VDFs is equal to $19 T_{\mathrm{Le}}$ (where $T_{\mathrm{Le}}$ is the Larmor period of electrons in the upstream region); it corresponds to a $30 \mathrm{~ms}$ time resolution, which is compatible with the MMS measurements of electron VDFs in a magnetic field of $23 \mathrm{nT}$.

The virtual satellite probes the jet's lateral edge, deflected towards the $-\boldsymbol{B} \times \nabla B$ direction in the vicinity of the TD (which could correspond to an idealized magnetopause). It moves within the energy-dispersed structure and detects the crescent-shaped electron VDFs illustrated in Fig. 5a-e. The morphology and orientation of the electron VDFs change in time. At $t_{1}=1.03 T_{\mathrm{Li}}$ (see Fig. 5a), the crescent is thinner and the phase density is enhanced in the $v_{x}>0$ sector of the perpendicular velocity space (as in Fig. $2 \mathrm{k}$ ). Up to $t_{5}=3.08 T_{\mathrm{Li}}$ (see Fig. 5e), the crescent becomes thicker and thicker and it rotates counter clockwise towards the $v_{y}>0$ sector, as the virtual satellite samples different areas close to the jet's 


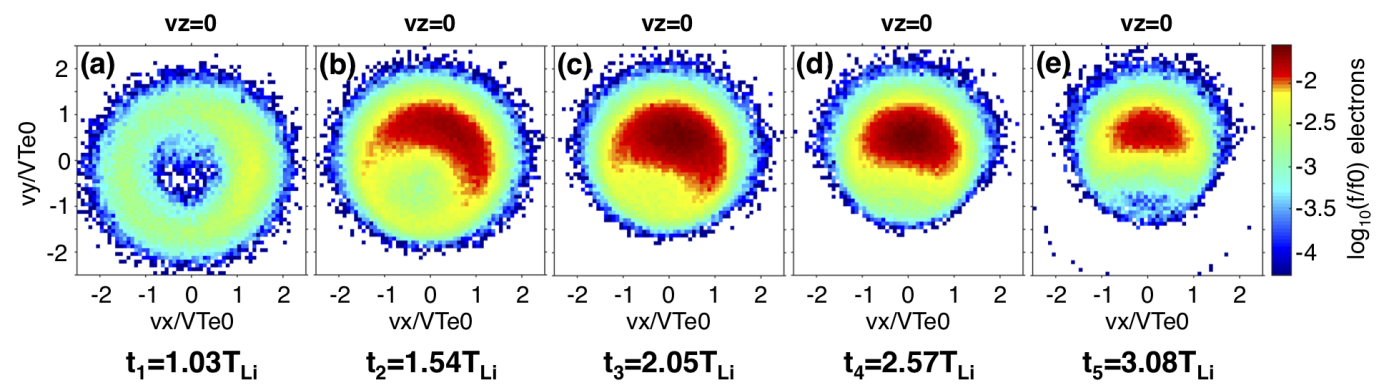

Figure 5. Virtual time series of five electron VDFs "measured" by a virtual satellite launched into the simulation domain. We illustrate the space of perpendicular velocities for $v_{z}=0$. The colour scale used is logarithmic.

boundaries. In a frame of reference attached to the deflected plasma jet, the satellite is moving away from the jet's edge at the lowest $y$ value, drifting along the positive direction of the $y$ axis and at the same time closer to the TD's surface. Indeed, at time $t_{1}$, the area sampled by the virtual satellite is similar to that of bin A9 in Fig. 2a, while at time $t_{5}$ the satellite is in a similar position to the centre of the bin A5 in Fig. 2a. Thus, the position of the satellite changes with respect to the plasma jet's core. At time $t_{1}$, the satellite is at its farthest distance from the jet's core along the $-\boldsymbol{B} \times \nabla B$ direction and the corresponding VDF has the largest central cavity in the space of perpendicular velocities. Later on, the satellite moves closer to the jet's core and the size of the VDF's central cavity decreases. The position of the satellite also changes with respect to the local orientation of the jet's boundaries. While at time $t_{1}$ most of the electrons are coming from above the satellite's sampling area (like the blue orbit in Fig. 4g), at $t_{5}$ most of them are coming from the left side (like the red orbit in Fig. 4g). Consequently, the peak of the gyrophase density in the space of perpendicular velocities rotates from the $v_{x}>0$ sector in Fig. 5a towards the $v_{y}>0$ sector in Fig. 5e. Since the properties of the measured electron VDFs depend on the relative location of the satellite with respect to the jet's core and boundaries, such velocity distribution functions could be used to estimate some geometrical and morphological features of a deflected plasma jet.

\section{Summary and conclusions}

Three-dimensional PIC simulations of the interaction between a low-beta plasma jet and a thin, steep and impenetrable tangential discontinuity with no magnetic shear emphasize the formation of crescent-shaped electron velocity distribution functions at the jet's boundaries. The simulation setup corresponds to an idealized, yet relevant, magnetic configuration likely to be observed at the frontside magnetopause during northward IMF. The results of our simulations show the following:
1. The crescent-shaped electron VDFs are observed in a magnetic field configuration that has no $\mathrm{X}$ line. Their occurrence and characteristics depend on the dynamic and geometric properties of the jet, as well as on the profile of the non-uniform magnetic and electric fields at the discontinuity.

2. The energy-dependent gradient-B drift of the electrons in the frontside of the jet leads to the formation of an energy-dispersed structure with ring-shaped velocity distribution functions: the electrons with higher gyration energies accumulate towards the jet's lateral edge along the $-\boldsymbol{B} \times \nabla \boldsymbol{B}$ direction, where the VDF shows a deficit of low energy electrons in the perpendicular velocity space.

3. Within the most lateral edges of the energy-dispersed structure, the ring of the VDF is incomplete and the VDF's shape changes from ring-shaped to crescentshaped. The crescent is due to the spatial anisotropy at the sharp edges of the plasma jet. It is produced by the remote sensing of high-energy particles whose guiding centres are localized inside the jet, as illustrated schematically in Fig. 4.

4. The crescent-shaped VDFs are produced simultaneously by (i) the velocity filtering effect of the gradient-B drift and (ii) the remote sensing of large Larmor radius electrons at the boundaries of the plasma jet.

5. Virtual time series of electron VDFs recorded by a virtual satellite launched into the simulation domain show that the ring-shaped and crescent-shaped electron VDFs are persistent and carried by the jet during its motion after interacting with the magnetic discontinuity. Their properties (like the size and position of the crescent) change with the satellite's location within the jet, and thus such VDFs can help to estimate the geometrical and morphological features of a deflected jet during or after its interaction with a tangential discontinuity or regions of sharp magnetic field variation. 
The new and original result of our study is the identification of crescent-shaped electron VDFs as a boundary effect at the edges of a finite-size three-dimensional plasma jet impacting on a tangential discontinuity with no field reversal. The properties of the crescents vary with the distance from the jet's centre in the $-\boldsymbol{B} \times \nabla B$ direction; they also depend on the local orientation of the jet's boundaries. The physical mechanism we propose to explain the crescent-shaped VDFs is based on finite Larmor radius effects. To our knowledge, this is for the first time that such electron velocity distributions have been emphasized in the absence of $X$ lines and magnetic field reversal, and the physical process proposed adds to the ones discussed in the past like, for instance, the remote sensing of a thin Harris sheet (Lee et al., 2004; Voitcu and Echim, 2012), the dynamics of non-magnetized Speiser-like electrons (Bessho et al., 2016; Shay et al., 2016; Lapenta et al., 2017) or the dynamics of magnetized electrons (Egedal et al., 2016) in the diffusion region of magnetic reconnection sites.

The crescent-shaped electron VDFs discussed in this paper and the ones reported in connection with magnetic reconnection at the dayside magnetopause show similar features in the perpendicular velocity space, but there are also significant differences between them related to the actual geometry of the physical processes leading to their formation. In our simulations, the electron crescents are obtained in connection to a counter-streaming jetting structure in the perpendicular direction to both the background magnetic field and the normal to the TD's surface (our simplified magnetopause) and involve velocity filtering and remote sensing effects; the corresponding VDFs are found inside and also in the vicinity of the discontinuity towards the $-\boldsymbol{B} \times \nabla B$ di- rection. On the other hand, in Bessho et al. (2016), Burch et al. (2016), Egedal et al. (2016), Shay et al. (2016) and Lapenta et al. (2017) for example, the electron crescents are obtained in connection to the anti-parallel jetting along the magnetospheric field direction produced by magnetic reconnection; the corresponding VDFs are found close to the magnetic null region. Egedal et al. (2016) show that the crescentshaped electron VDFs are formed along the low-density separatrix in a thin boundary layer with the width of approximately one electron Larmor radius. In our simulations, the crescent-shaped electron VDFs are observed over a wide area, covering a few tens of electron Larmor radii in the perpendicular plane to the magnetic field.

The simulations discussed in this paper provide new insight into the microphysics of plasma-field interaction near magnetic boundaries and contribute to advancing the understanding of the dynamics and kinetics of magnetosheath high-speed jets streaming towards the magnetopause during periods of northward IMF. We also demonstrate that the crescent is a persistent feature of the VDFs that can be detected at relatively large distances from the interaction with the discontinuity and therefore might be used as the kinetic signature of such an interaction.

Data availability. The simulation data used to produce all the plots and analyses included in this paper can be requested by sending an e-mail to Gabriel Voitcu at one of the following addresses: gabi@spacescience.ro or gabriel.voitcu@gmail.com. 


\section{Appendix A}

We present here the full map of the electron velocity distribution functions for all the spatial bins defined in the $x O y$ plane, perpendicular to the magnetic field direction. In Fig. A1 we show the electron number density in the plane $x O y$ for $z=403$, at two instances, (a) $t=1 T_{\mathrm{Li}}$ and (b) $t=1.9 T_{\mathrm{Li}}$, together with all the bins used to calculate the electron VDFs. There are 88 bins in Fig. A1a and 84 bins in Fig. A1b that cover the entire plasma jet at the two different time instances. The VDFs for each spatial bin are illustrated in Figs. A2 (at $\left.t=1 T_{\mathrm{Li}}\right)$ and $\mathrm{A} 3$ (at $t=1.9 T_{\mathrm{Li}}$ ) where we show cross sections corresponding to $v_{z}=0$. The nine selected bins A $1-\mathrm{A} 9$ in Fig. 2 of the article correspond to bins 39, 10, 21, 43, 65, 76, 87, 75 and 86 in Fig. A1a. The nine selected bins B1-B9 in Fig. 3 of the article correspond to bins 39, 6, 13, 41, 69, 76, 83, 75 and 82 in Fig. A1b. One note that in the bulk of the jet the VDF is a displaced Maxwellian, consistent with the forward motion of the plasma. The non-gyrotropic effects are localized at the edges of the plasma jet.
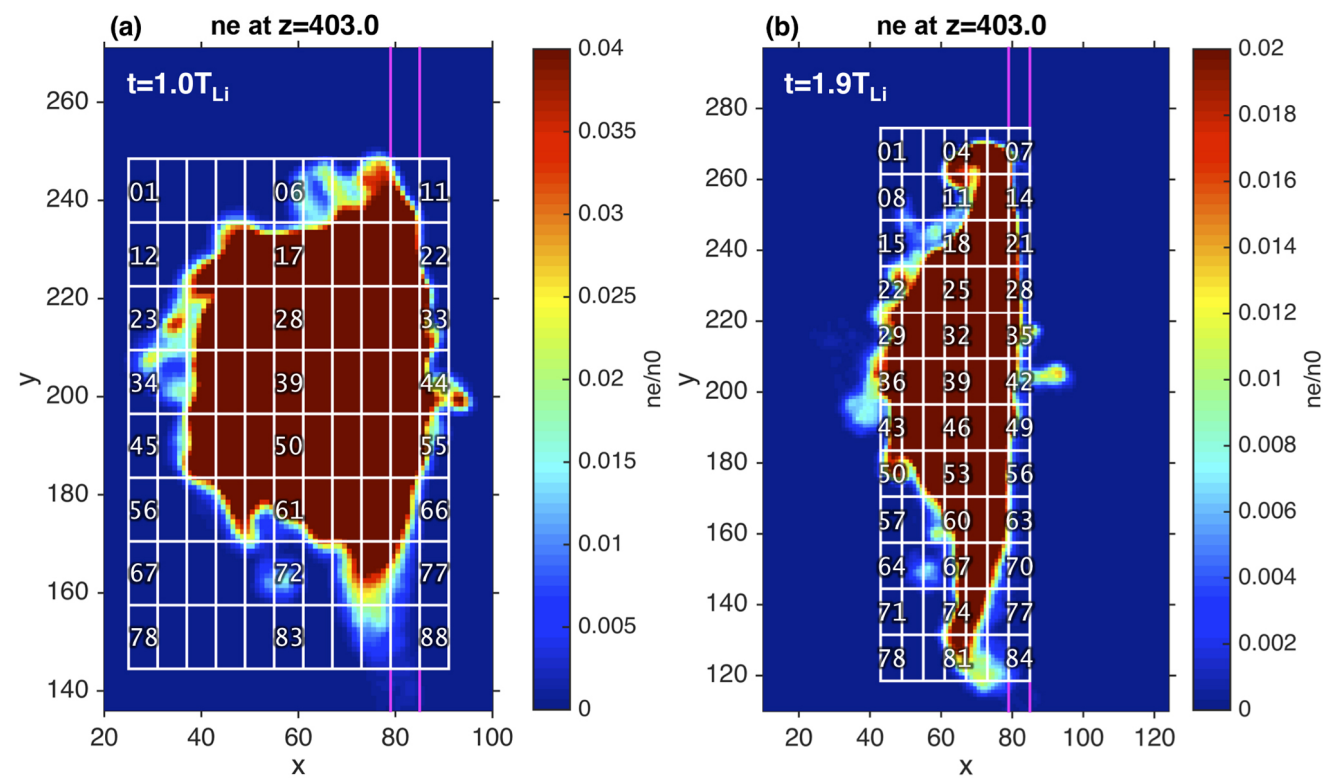

Figure A1. Electron number density for the $z=403$ cross section together with the entire spatial grid used to calculate the electron velocity distribution functions, at (a) $t=1 T_{\mathrm{Li}}$ and (b) $t=1.9 T_{\mathrm{Li}}$. There are 88 spatial bins in panel (a) and 84 in (b). 


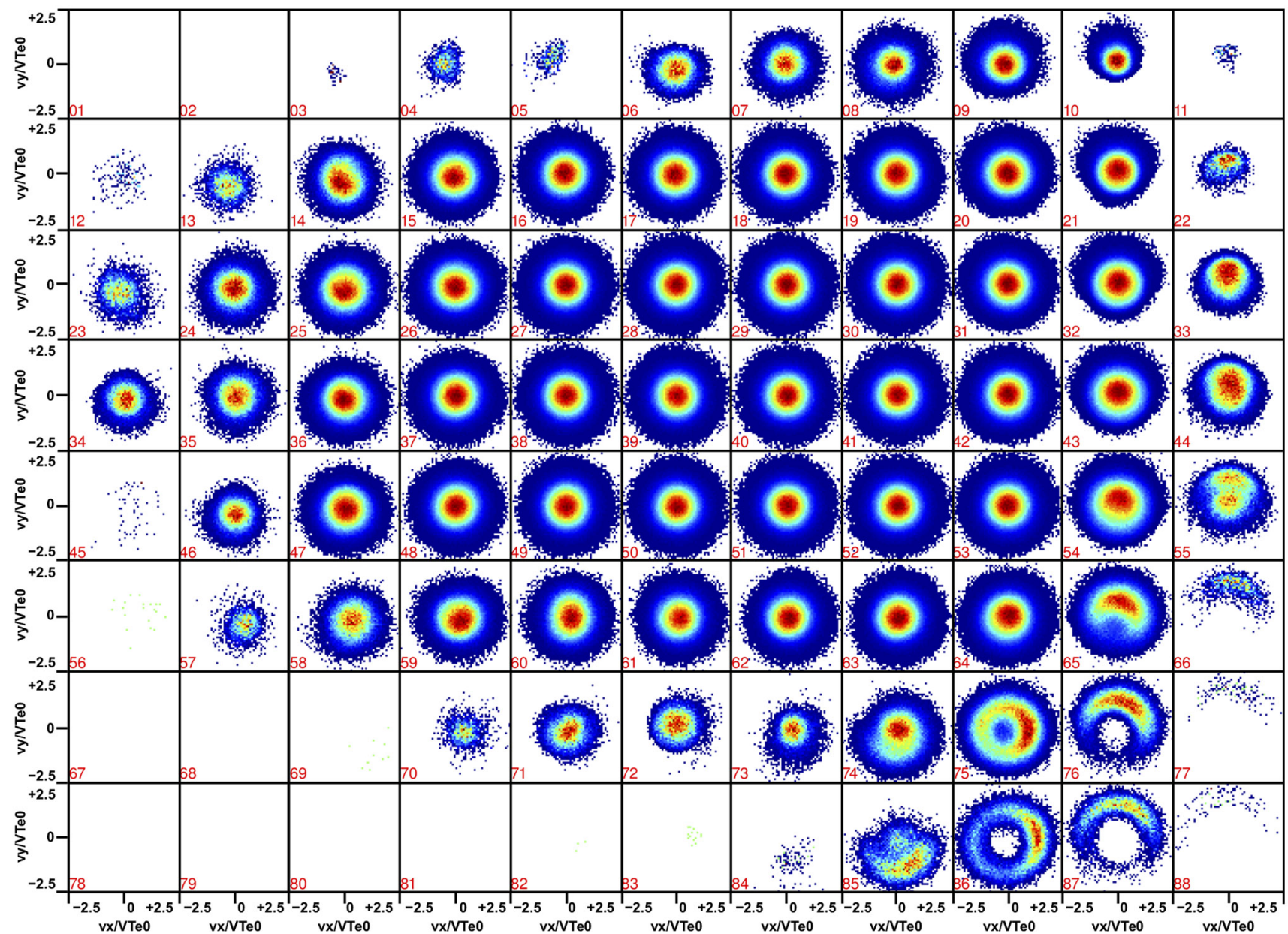

Figure A2. Electron VDFs, at $t=1 T_{\mathrm{Li}}$, for each of the 88 spatial bins defined in Fig. A1a. We show $v_{z}=0$ cross sections. The nine VDFs presented in Fig. 2 of the article correspond to bins 39, 10, 21, 43, 65, 76, 87, 75 and 86. 


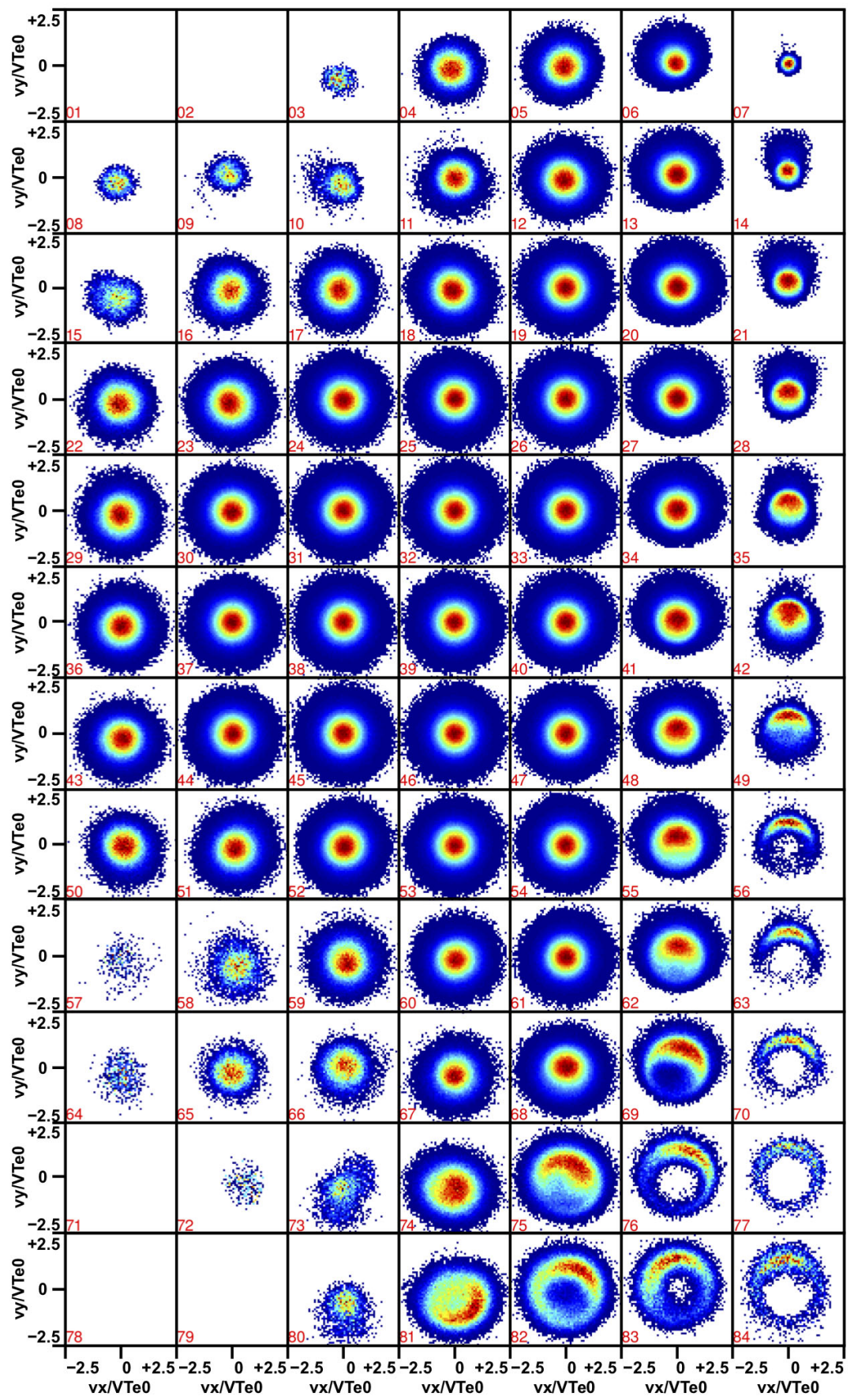

Figure A3. Electron VDFs, at $t=1.9 T_{\mathrm{Li}}$, for each of the 84 spatial bins defined in Fig. A1b. We show $v_{z}=0$ cross sections. The nine VDFs presented in Fig. 3 of the article correspond to bins 39, 6, 13, 41, 69, 76, 83, 75 and 82. 
Author contributions. GV: conceptualization, methodology, software, formal analysis, investigation, visualization, writing - original draft and review and editing. ME: conceptualization, methodology, investigation, supervision, visualization, writing - original draft and review and editing.

Competing interests. The authors declare that they have no conflict of interest.

Acknowledgements. The authors acknowledge support from the Romanian Space Agency (ROSA) through the Space Technology and Advanced Research (STAR) Programme under contracts $182 / 2017$ (OANA) and 122/2017 (ODYN) and also from the Romanian Executive Agency for Higher Education, Research, Development and Innovation Funding (UEFISCDI) through project VESS/2018. Marius Echim acknowledges support from the Belgian Solar-Terrestrial Centre of Excellence (STCE) in Brussels, Belgium.

Edited by: Elias Roussos

Reviewed by: two anonymous referees

\section{References}

Archer, M. O. and Horbury, T. S.: Magnetosheath dynamic pressure enhancements: occurrence and typical properties, Ann. Geophys., 31, 319-331, https://doi.org/10.5194/angeo-31-319-2013, 2013.

Bessho, N., Chen, L.-J., and Hesse, M.: Electron distribution functions in the diffusion region of asymmetric magnetic reconnection, Geophys. Res. Lett., 43, 1828-1836, https://doi.org/10.1002/2016GL067886, 2016.

Buneman, O.: TRISTAN - The 3D electromagnetic particle code, in: Computer Space Plasma Physics: Simulation Techniques and Software, edited by: Matsumoto, H. and Omura, Y., Terra Scientific Publishing Company, Tokyo, 1993.

Burch, J. L., Torbert, R. B., Phan, T. D., Chen, L.-J., Moore, T. E., Ergun, R. E., Eastwood, J. P., Gershman, D. J., Cassak, P. A., Argall, M. R., Wang, S., Hesse, M., Pollock, C. J., Giles, B. L., Nakamura, R., Mauk, B. H., Fuselier, S. A., Russell, C. T., Strangeway, R. J., Drake, J. F., Shay, M. A., Khotyaintsev, Yu. V., Lindqvist, P.-A., Marklund, G., Wilder, F. D., Young, D. T., Torkar, K., Goldstein, J., Dorelli, J. C., Avanov, L. A., Oka, M., Baker, D. N., Jaynes, A. N., Goodrich, K. A., Cohen, I. J., Turner, D. L., Fennell, J. F., Blake, J. B., Clemmons, J., Goldman, M., Newman, D., Petrinec, S. M., Trattner, K. J., Lavraud, B., Reiff, P. H., Baumjohann, W., Magnes, W., Steller, M., Lewis, W., Saito, Y., Coffey, V., and Chandler, M.: Electronscale measurements of magnetic reconnection in space, Science, 352, 2939, https://doi.org/10.1126/science.aaf2939, 2016.

Cai, D. S. and Buneman, O.: Formation and stability of polarization sheaths of a cross-field beam, Phys. Fluids B-Plasma, 4, 10331046, 1992.

Chen, F. F.: Introduction to plasma physics and controlled fusion, 2nd Edn., Plenum Press, New York, 1974.
Dai, W. and Woodward, P. R.: Two-dimensional simulations for the impulsive penetration of a solar wind filament into the magnetosphere, J. Geophys. Res., 99, 8577-8584, 1994.

Dai, W. and Woodward, P. R.: Interactions between a solar wind filament and an open magnetosphere, J. Geophys. Res., 100, 14843-14852, 1995.

Dai, W. and Woodward, P. R.: Oblique penetration of solar-wind filaments into the magnetosphere, J. Plasma Phys., 60, 711-729, 1998.

Dmitriev, A. V. and Suvorova, A. V.: Traveling magnetopause distortion related to a large-scale magnetosheath plasma jet: THEMIS and ground-based observations, J. Geophys. Res., 117, A08217, https://doi.org/10.1029/2011JA016861, 2012.

Dmitriev, A. V. and Suvorova, A. V.: Large-scale jets in the magnetosheath and plasma penetration across the magnetopause: THEMIS observations, J. Geophys. Res.-Space, 120, 44234437, https://doi.org/10.1002/2014JA020953, 2015.

Echim, M. M. and Lemaire, J. F.: Laboratory and numerical simulations of the impulsive penetration mechanism, Space Sci. Rev., 92, 565-601, 2000.

Echim, M. M. and Lemaire, J.: Two-dimensional Vlasov solution for a collisionless plasma jet across transverse magnetic field lines with a sheared bulk velocity, Phys. Rev. E, 72, 036405, https://doi.org/10.1103/PhysRevE.72.036405, 2005.

Egedal, J., Le, A., Daughton, W., Wetherton, B., Cassak, P. A., Chen, L.-J., Lavraud, B., Torbert, R. B., Dorelli, J., Gershman, D. J., and Avanov, L. A.: Spacecraft Observations and Analytic Theory of Crescent-Shaped Electron Distributions in Asymmetric Magnetic Reconnection, Phys. Rev. Lett., 117, 185101, https://doi.org/10.1103/PhysRevLett.117.185101, 2016.

Galvez, M.: Computer simulation of a plasma streaming across a magnetic field, Phys. Fluids, 30, 2729-2739, 1987.

Galvez, M. and Borovsky, J. E.: The expansion of polarization charge layers into a magnetized vacuum: theory and computer simulations, Phys. Fluids B-Plasma, 3, 1892-1907, 1991.

Galvez, M., Gary, S. P., Barnes, C., and Winske, D.: Computer simulations of plasma expansion across a magnetic field, Phys. Fluids, 31, 1554-1567, 1988.

Gunell, H., Walker, J. J., Koepke, M. E., Hurtig, T., Brenning, N., and Nilsson, H.: Numerical experiments on plasmoids entering a transverse magnetic field, Phys. Plasmas, 16, 112901, https://doi.org/10.1063/1.3267860, 2009.

Gunell, H., Nilsson, H., Stenberg, G., Hamrin, M., Karlsson, T., Maggiolo, R., André, M., Lundin, R., and Dandouras, I.: Plasma penetration of the dayside magnetopause, Phys. Plasmas, 19, 072906, https://doi.org/10.1063/1.4739446, 2012.

Gunell, H., Stenberg Wieser, G., Mella, M., Maggiolo, R., Nilsson, H., Darrouzet, F., Hamrin, M., Karlsson, T., Brenning, N., De Keyser, J., André, M., and Dandouras, I.: Waves in high-speed plasmoids in the magnetosheath and at the magnetopause, Ann. Geophys., 32, 991-1009, https://doi.org/10.5194/angeo-32-9912014, 2014.

Haaland, S. E., Sonnerup, B. U. Ö., Dunlop, M. W., Balogh, A., Georgescu, E., Hasegawa, H., Klecker, B., Paschmann, G., PuhlQuinn, P., Rème, H., Vaith, H., and Vaivads, A.: Four-spacecraft determination of magnetopause orientation, motion and thickness: comparison with results from single-spacecraft methods, Ann. Geophys., 22, 1347-1365, https://doi.org/10.5194/angeo22-1347-2004, 2004. 
Hietala, H., Partamies, N., Laitinen, T. V., Clausen, L. B. N., Facskó, G., Vaivads, A., Koskinen, H. E. J., Dandouras, I., Rème, H., and Lucek, E. A.: Supermagnetosonic subsolar magnetosheath jets and their effects: from the solar wind to the ionospheric convection, Ann. Geophys., 30, 33-48, https://doi.org/10.5194/angeo30-33-2012, 2012.

Hietala, H., Phan, T. D., Angelopoulos, V., Oieroset, M., Archer, M. O., Karlsson, T., and Plaschke, F.: In situ observations of a magnetosheath high-speed jet triggering magnetopause reconnection, Geophys. Res. Lett., 45, 1732-1740, https://doi.org/10.1002/2017GL076525, 2018.

Huba, J. D.: Impulsive plasmoid penetration of a tangential discontinuity: Two-dimensional ideal and Hall magnetohydrodynamics, J. Geophys. Res., 101, 24855-24868, 1996.

Hurtig, T., Brenning, N., and Raadu, M. A.: Three-dimensional electrostatic particle-in-cell simulation with open boundaries applied to a plasma beam entering a curved magnetic field, Phys. Plasmas, 10, 4291-4305, 2003.

Karlsson, T., Brenning, N., Nilsson, H., Trotignon, J.-G., Vallières, X., and Facsko, G.: Localized density enhancements in the magnetosheath: Three-dimensional morphology and possible importance for impulsive penetration, J. Geophys. Res., 117, A03227, https://doi.org/10.1029/2011JA017059, 2012.

Karlsson, T., Plaschke, F., Hietala, H., Archer, M., BlancoCano, X., Kajdic, P., Lindqvist, P.-A., Marklund, G., and Gershman, D. J.: Investigating the anatomy of magnetosheath jets - MMS observations, Ann. Geophys., 36, 655-677, https://doi.org/10.5194/angeo-36-655-2018, 2018.

Lapenta, G., Berchem, J., Zhou, M., Walker, R. J., El-Alaoui, M., Goldstein, M. L., Paterson, W. R., Giles, B. L., Pollock, C. J., Russell, C. T., Strangeway, R. J., Ergun, R. E., Khotyaintsev, Y. V., Torbert, R. B., and Burch, J. L.: On the origin of the crescent-shaped distributions observed by MMS at the magnetopause, J. Geophys. Res.-Space, 122, 2024-2039, https://doi.org/10.1002/2016JA023290, 2017.

Lee, E., Wilber, M., Parks, G. K., Min, K. W., and Lee, D.-Y.: Modeling of remote sensing of thin current sheet, Geophys. Res. Lett., 31, L21806, https://doi.org/10.1029/2004GL020331, 2004.

Lemaire, J.: Impulsive penetration of filamentary plasma elements into the magnetospheres of the Earth and Jupiter, Planet. Space Sci., 25, 887-890, 1977.

Lemaire, J.: Plasmoid motion across a tangential discontinuity (with applications to the magnetopause), J. Plasma Phys., 33, 425-436, 1985.

Lemaire, J. and Roth, M.: Non-steady-state solar windmagnetosphere interaction, Space Sci. Rev., 57, 59-108, https://doi.org/10.1007/BF00195951, 1991.

Livesey, W. A. and Pritchett, P. L.: Two-dimensional simulations of a charge-neutral plasma beam injected into a transverse magnetic-field, Phys. Fluids B-Plasma, 1, 914-922, 1989.

Lu, G., Onsager, T. G., Le, G., and Russell, C. T.: Ion injections and magnetic field oscillations near the highlatitude magnetopause associated with solar wind dynamic pressure enhancement, J. Geophys. Res., 109, A06208, https://doi.org/10.1029/2003JA010297, 2004.

Lundin, R. and Aparicio, B.: Observations of penetrated solar wind plasma elements in the plasma mantle, Planet. Space Sci., 30, 81-91, 1982.
Lyatsky, W., Pollock, C., Goldstein, M. L., Lyatskaya, S., and Avanov, L.: Penetration of magnetosheath plasma into dayside magnetosphere: 1. Density, velocity, and rotation, J. Geophys. Res.-Space, 121, 7699-7712, https://doi.org/10.1002/2015JA022119, 2016.

Ma, Z. V., Hawkins, J. G., and Lee, L. C.: A simulation study of impulsive penetration of solar wind irregularities into the magnetosphere at the dayside magnetopause, J. Geophys. Res., 96, 15751-15765, 1991.

Marcucci, M. F., Bavassano Cattaneo, M. B., Pallocchia, G., Amata, E., Bruno, R., Di Lellis, A. M., Formisano, V., Rème, H., Bosqued, J. M., Dandouras, I., Sauvaud, J.-A., Kistler, L. M., Moebius, E., Klecker, B., Carlson, C. W., Parks, G. K., McCarthy, M., Korth, A., Lundin, R., and Balogh, A.: Energetic magnetospheric oxygen in the magnetosheath and its response to IMF orientation: Cluster observations, J. Geophys. Res., 109, A07203, https://doi.org/10.1029/2003JA010312, 2004.

Nakamura, M., Paschmann, G., Baumjohann, W., and Sckopke, N.: Ion distributions and flows near the neutral sheet, J. Geophys. Res., 96, 5631-5649, https://doi.org/10.1029/90JA02495, 1991.

Nakamura, M., Paschmann, G., Baumjohann, W., and Sckopke, N.: Ion distributions and flows in and near the plasma sheet boundary layer, J. Geophys. Res. 97, 1449-1460, https://doi.org/10.1029/91JA02361, 1992.

Neubert, T., Miller, R. H., Buneman, O., and Nishikawa, K. I.: The dynamics of low-beta plasma clouds as simulated by a 3dimensional, electromagnetic particle code, J. Geophys. Res.Space, 97, 12057-12072, 1992.

Northrop, T. G.: Adiabatic charged-particle motion, Rev. Geophys., 1, 283-304, https://doi.org/10.1029/RG001i003p00283, 1963.

Palmroth, M., Hietala, H., Plaschke, F., Archer, M., Karlsson, T., Blanco-Cano, X., Sibeck, D., Kajdic, P., Ganse, U., Pfau-Kempf, Y., Battarbee, M., and Turc, L.: Magnetosheath jet properties and evolution as determined by a global hybrid-Vlasov simulation, Ann. Geophys., 36, 1171-1182, https://doi.org/10.5194/angeo36-1171-2018, 2018.

Plaschke, F., Glassmeier, K.-H., Auster, H. U., Constantinescu, O. D., Magnes, W., Angelopoulos, V., Sibeck, D. G., and McFadden, J. P.: Standing Alfven waves at the magnetopause, Geophys. Res. Lett., 36, L02104, https://doi.org/10.1029/2008GL036411, 2009.

Plaschke, F., Hietala, H., and Angelopoulos, V.: Anti-sunward highspeed jets in the subsolar magnetosheath, Ann. Geophys., 31, 1877-1889, https://doi.org/10.5194/angeo-31-1877-2013, 2013.

Plaschke, F., Hietala, H., Angelopoulos, V., and Nakamura, R.: Geoeffective jets impacting the magnetopause are very common, J. Geophys. Res.-Space, 121, 3240-3253, https://doi.org/10.1002/2016JA022534, 2016.

Plaschke, F., Karlsson, T., Hietala, H., Archer, M., Vö rös, Z., Nakamura, R., Magnes, W., Baumjohann, W., Torbert, R. B., Russell, C. T., Giles, B. L.: Magnetosheath highspeed jets: Internal structure and interaction with ambient plasma, J. Geophys. Res.-Space, 122, 10157-10175, https://doi.org/10.1002/2017JA024471, 2017.

Roth, M., DeKeyser, J., and Kuznetsova, M. M.: Vlasov theory of the equilibrium structure of tangential discontinuities in space plasmas, Space Sci. Rev., 76, 251-317, https://doi.org/10.1007/BF00197842, 1996. 
Savin, S., Amata, E., Zelenyi, L., Lutsenko, V., Safrankova, J., Nemecek, Z., Borodkova, N., Buechner, J., Daly, P. W., Kronberg, E. A., Blecki, J., Budaev, V., Kozak, L., Skalsky, A., and Lezhen, L.: Super fast plasma streams as drivers of transient and anomalous magnetospheric dynamics, Ann. Geophys., 30, 1-7, https://doi.org/10.5194/angeo-30-1-2012, 2012.

Savoini, P., Scholer, M., and Fujimoto, M.: Two-dimensional hybrid simulations of impulsive plasma penetration through a tangential discontinuity, J. Geophys. Res., 99, 19377-19391, 1994.

Schmidt, G.: Plasma motions across magnetic fields, Phys. Fluids, 3, 961-965, 1960.

Shay, M. A., Phan, T. D., Haggerty, C. C., Fujimoto, M., Drake, J. F., Malakit, K., Cassak, P. A., and Swisdak, M.: Kinetic signatures of the region surrounding the $\mathrm{X}$ line in asymmetric (magnetopause) reconnection, Geophys. Res. Lett., 43, 4145-4154, https://doi.org/10.1002/2016GL069034, 2016.

Shi, Q. Q., Zhong, Q.-G., Fu, S. Y., Dunlop, M. W., Pu., Z. Y., Parks, G. K., Wei, Y., Li, W. H., Zhang, H., Nowada, M., Wang, Y. B., Sun, W. J., Xiao, T., Reme, H., Carr, C., Fazakerley, A., and Lucek, E.: Solar wind entry into the high-latitude terrestrial magnetosphere during geomagnetically quiet times, Nat. Commun., 4, 1466, https://doi.org/10.1038/ncomms2476, 2013.

Voitcu, G.: Kinetic simulations of plasma dynamics across magnetic fields and applications to the physics of planetary magnetospheres, $\mathrm{PhD}$ thesis, Doctoral School of Physics, University of Bucharest, Romania, 2014.

Voitcu, G. and Echim, M.: Ring-shaped velocity distribution functions in energy-dispersed structures formed at the boundaries of a proton stream injected into a transverse magnetic field: Test-kinetic results, Phys. Plasmas, 19, 022903, https://doi.org/10.1063/1.3686134, 2012.
Voitcu, G. and Echim, M.: Transport and entry of plasma clouds/jets across transverse magnetic discontinuities: Three-dimensional electromagnetic particle-in-cell simulations, J. Geophys. Res.Space, 121, 4343-4361, https://doi.org/10.1002/2015JA021973, 2016.

Voitcu, G. and Echim, M.: Tangential deflection and formation of counterstreaming flows at the impact of a plasma jet on a tangential discontinuity, Geophys. Res. Lett., 44, 5920-5927, https://doi.org/10.1002/2017GL073763, 2017.

Voitcu, G., Echim, M., and Marchand, R.: Comparative study of forward and backward test-kinetic simulation approaches, Comput. Phys. Commun., 183, 2561-2569, https://doi.org/10.1016/j.cpc.2012.07.005, 2012.

Wilber, M., Lee, E., Parks, G. K., Meziane, K., Carlson, C. W., McFadden, J. P., Rème, H, Dandouras, I., Sauvaud, J.-A., Bosqued, J.-M., Kistler, L., Möbius, E., McCarthy, M., Korth, A., Klecker, B., Bavassano-Cattaneo, M.-B., Lundin, R., and Lucek, E.: Cluster observations of velocity space-restricted ion distributions near the plasma sheet, Geophys. Res. Lett., 31, L24802, https://doi.org/10.1029/2004GL020265, 2004.

Willis, D. M.: The magnetopause: Microstructure and interaction with magnetospheric plasma, J. Atmos. Terr. Phys., 40, 301-322, https://doi.org/10.1016/0021-9169(78)90047-8, 1978.

Woch, J. and Lundin, R.: Temporal magnetosheath plasma injection observed with Viking: a case study, Ann. Geophys., 9, 133-142, 1991.

Woch, J. and Lundin, R.: Signatures of transient boundary layer processes observed with Viking, J. Geophys. Res., 97, 1431-1447, https://doi.org/10.1029/91JA02490, 1992.

Yamauchi, M., Woch, J., Lundin, R., Shapshak, M., and Elphinstone, R.: A new type of ion injection event observed by Viking, Geophys. Res. Lett., 20, 795-798, https://doi.org/10.1029/93GL00855, 1993. 